\title{
Novel sensing approach for LPG leakage detection: Part II: Effects of particle size, composition and coating layer thickness
}

\author{
Anindya Nag, Asif I. Zia, Li Xie, Subhas Chandra \\ Mukhopadhyay \\ School of Engineering and Advanced Technology \\ Massey University (Manawatu Campus) \\ Palmerston North, New Zealand
}

\author{
Jürgen Kosel \\ Sensing, Magnetism and Microsystems Group, \\ King Abdullah University of Science and Technology, \\ Saudi Arabia
}

\begin{abstract}
Prominent research has been going on to develop a low-cost, efficient gas sensing system. The paper presents a continuation of our earlier research work done to develop a new sensing approach for gas detection at ambient conditions. The work exhibits the optimization of the response time of the sensor by inhabiting characteristic changes like variation in the concentration of the dispersion medium, thickness of the coating and the size of the dispersed medium. Different concentrations of the dispersion medium in the coated suspension were tested to determine the optimal composition required to achieve the highest sensitivity of the tin oxide $\left(\mathrm{SnO}_{2}\right)$ layer towards the tested gas. The control over adsorption and desorption of the gas molecules in the coated layer was achieved by investigating the particle size of the dispersed medium. The response time of the coated sensor was encouraging and owns a promising potential to the development of a more efficient gas sensing system.
\end{abstract}

Keywords-Interdigital sensors, LPG, electrochemical impedance spectroscopy, $\mathrm{SnO}_{2}$, coating, dispersion medium, sieving.

\section{INTRODUCTION}

Liquefied Petroleum Gas (LPG) detection is one of the most important areas to be researched taking into account the different sectors it is used in the society. The most common use among them is in the domestic homes as a cooking gas. Due to its combustible nature, the leakage of this gas needs to be at a minimum level to avoid accidents $[1,2]$ and maintain the air quality [3, 4]. In general cases, they are sealed in pressurized bottles.

Research regarding the sensorial gas detection has been going for quite some time [5]. All of them are based on the idea of inducing a heating coil inside the electrochemical cell to increase the surface temperature of the sensor causing an increase in the sensitivity of the sensor. But the use of the heating element is disadvantageous in many ways like high power consumption and higher technical complexity. The system is also not practical to consider for real-time scenarios.

The paper was submitted on October 8, 2015.

The authors are with the School of Engineering and Advanced Technology, Massey University, Palmerston North, New Zealand. The email of the corresponding author is S.C.Mukhopadhyay@massey.ac.nz
Another popular method applied for sensing is doping increasing the conductivity [6-9]. Even though doping seems to a viable option, but the sensitivity of the sensor will gradually decrease once the extrinsic current caused due to the addition of dopants and intrinsic current within the sensor becomes equal. The addition of dopants is also not a cost effective process. A gas sensing system working in ambient conditions is therefore the state-of-art. The investigation on the detection of LPG in ambient temperature and humidity conditions is still unreported. The work in this paper is a continuance of the approach described in our previous paper, "Novel sensing approach for LPG leakage detection: Part I: Operating Mechanism and Preliminary Results.”. Different attributes of the coated layer is varied to optimize the response time of the sensor towards a specified concentration of the gas. Tin oxide $\left(\mathrm{SnO}_{2}\right)$ has been used in many investigations [7, 1013] for the detection of gases due to its semi-conductive property. The structure of $\mathrm{SnO}_{2}$ [14] makes it advantageous for conduction due to the availability of surface oxygen atoms. As described in our previous report, the sensing system was tested with different concentrations of LPG in ppm to verify the functionality of the system. Here, optimization of the response time is done by working on the characteristics of the thin film coated on the sensing surface. Electrochemical impedance spectroscopy [15-18] has been used to study the signal properties of the interdigital sensor [19-23].

The paper is divided into five sub-sections. Following the introduction given in section I, materials and methodology is described in section II. This part shows the SEM images of the coated sensor and the effect of duration of spin coating on it. Then the results of the gas sensing experiments are given in section III. The change on the response time due the concentration of dispersion medium and the size of dispersed particles are described in this section. Section IV provides an ideology of a microcontroller based gas sensing system with the fabricated circuit and some preliminary results. Section V gives the conclusion for the experiments conducted to validate the proposed novel approach.

\section{MATERIALS AND METHODOLOGY}

The spin coating of the suspension layer on the interdigital sensor was performed with three different durations, 15, 20 
and 25 seconds. Table 1 shows the average thickness of the three readings at different spin coating duration, each of which is an average of five readings taken vertically through an electron beam from different locations of the coated sensor. Figures 1-3 show the SEM images of the coated interdigital sensor. These images were obtained from a FEI Quanta 200 model with an accelerating voltage of $20 \mathrm{kV}$. It is seen from figure 1 that the amount of $\mathrm{SnO}_{2}$ present on the sensing surface of the sensor with 15 seconds of spin coating is too high. The layer is also uneven with the maximum proportion of the suspension layer cluttered on the middle of the sensing surface. The uniformity is somewhat maintained in the layer for a 20 seconds of spin coating as shown in figure 2 .

Table 1: Spin coating duration and their respective thickness

\begin{tabular}{|c|c|}
\hline $\begin{array}{c}\text { Spin coating duration } \\
\text { (seconds) }\end{array}$ & $\begin{array}{c}\text { Thickness of the layer } \\
\text { (microns) }\end{array}$ \\
\hline 15 & 2.33 \\
\hline 20 & 2.0475 \\
\hline 25 & 1.3675 \\
\hline
\end{tabular}

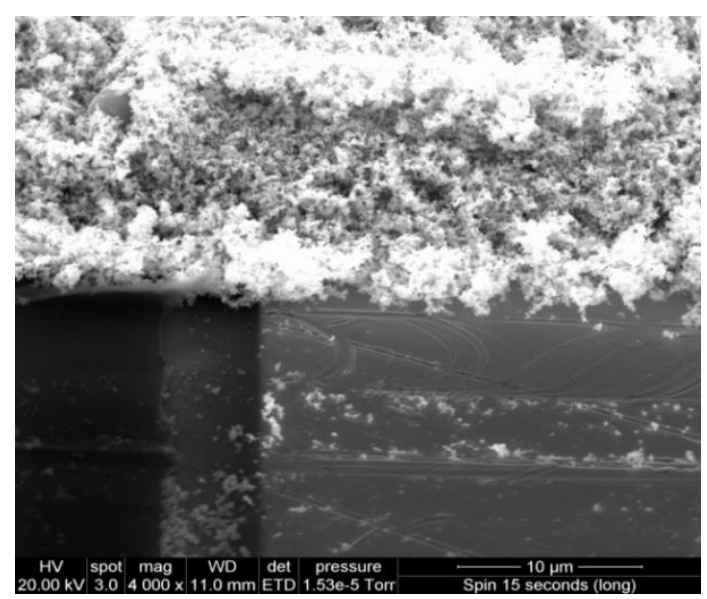

Fig. 1: SEM image of $\mathrm{SnO}_{2}$ coated layer for spin coating of 15 seconds.

From figure 3, the coated layer is seen to have pores in it, and the presence of $\mathrm{SnO}_{2}$ is also uneven in the layer. Thus, the uniformity of the coated layer is comparatively the optimum one for the duration of 20 seconds of spin coating. The operating frequency of $15 \mathrm{~Hz}$ was maintained through the experimental duration. The initial tests were done to determine the serial and parallel stray capacitances of LCR meter. The gas and air switches were turned on and off alternatively at an interval of 180 seconds. Readings of the response signal values were taken at a fixed interval of 2 seconds. Switching on and off of gas was done to calculate the change in a response time of the sensors. This cycle was repeated for a fixed duration to observe the sensitivity of the specified coated layer on the interdigital sensor.

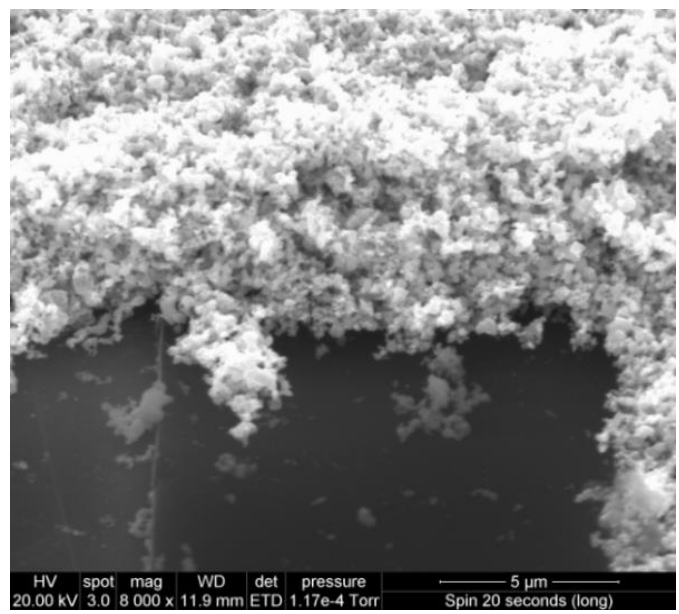

Fig. 2: SEM image of $\mathrm{SnO}_{2}$ coated layer for spin coating of 20 seconds

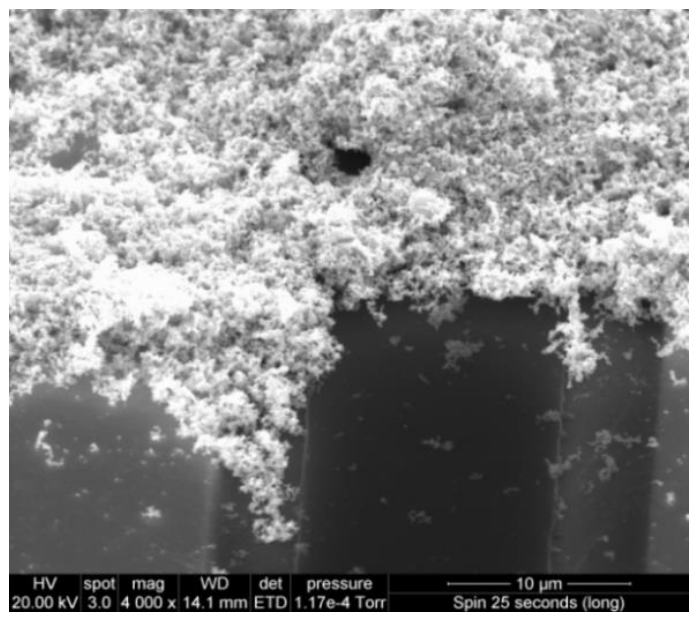

Fig. 3: SEM image of $\mathrm{SnO}_{2}$ coated layer for spin coating of 25 seconds.

\section{RESULTS AND DISCUSSIONS}

\subsection{Results}

The experiments were conducted using suspensions with $\mathrm{SnO}_{2}$ as the dispersed medium and using ethyl alcohol as the dispersion medium. A semi-solid porous suspension was formed on mixing the two mentioned compounds. Table 2 shows the concentrations of dispersed and dispersion medium mixed to form the coating layer. It is seen from the table, the dispersed medium had a fixed amount of 2 grams. So, the change in response of the coated interdigital sensors was determined with respect to different concentrations of the dispersion medium. The measured response signal was optimized by varying the particle size and coating thickness. Since the last paper of our gas sensing approach explained that the imaginary part of the impedance does not change much as compared to the real part; the change in the real part of the impedance is studied corresponding to the gas conditions. The blue line in the following experimental results denotes real part of the impedance of the sensor whereas the red one (square wave) denotes the condition of gas cycles. 
Table 2: Different concentrations of dispersion medium with dispersed phase used in the experiment

\begin{tabular}{|c|c|c|c|c|}
\hline Sieved particles & Suspension & $\begin{array}{c}\mathbf{S n O}_{2} \text { content } \\
(\text { grams })\end{array}$ & $\begin{array}{c}\text { Ethyl alcohol content } \\
(\mathbf{m l})\end{array}$ & $\begin{array}{c}\text { Size of SnO } \\
\text { (microns) }\end{array}$ \\
\hline- & Suspension 1 & 2 & 5.5 & - \\
\hline- & Suspension 2 & 2 & 6 & Between 75-63 \\
\hline Suspension 2.1 & - & 2 & 6 & Between 63-53 \\
\hline Suspension 2.2 & - & 2 & 6 & Between 53-45 \\
\hline Suspension 2.3 & - & 2 & 6 & Less than 45 \\
\hline Suspension 2.4 & - & 2 & 6.5 & - \\
\hline- & Suspension 3 & 2 & 7 & - \\
\hline- & Suspension 4 & 2 & 6 & \\
\hline
\end{tabular}

\section{Experiment with $2 \mathrm{gm} \mathrm{SnO}_{2}+5.5 \mathrm{ml} \mathrm{Et-OH}$}

The response of the interdigital sensor to LPG with a dispersion medium of $5.5 \mathrm{ml}$ of ethyl alcohol is shown in figure 4. The response time for the gas-on condition was around 40 seconds and for the gas-off condition was around 25 seconds.

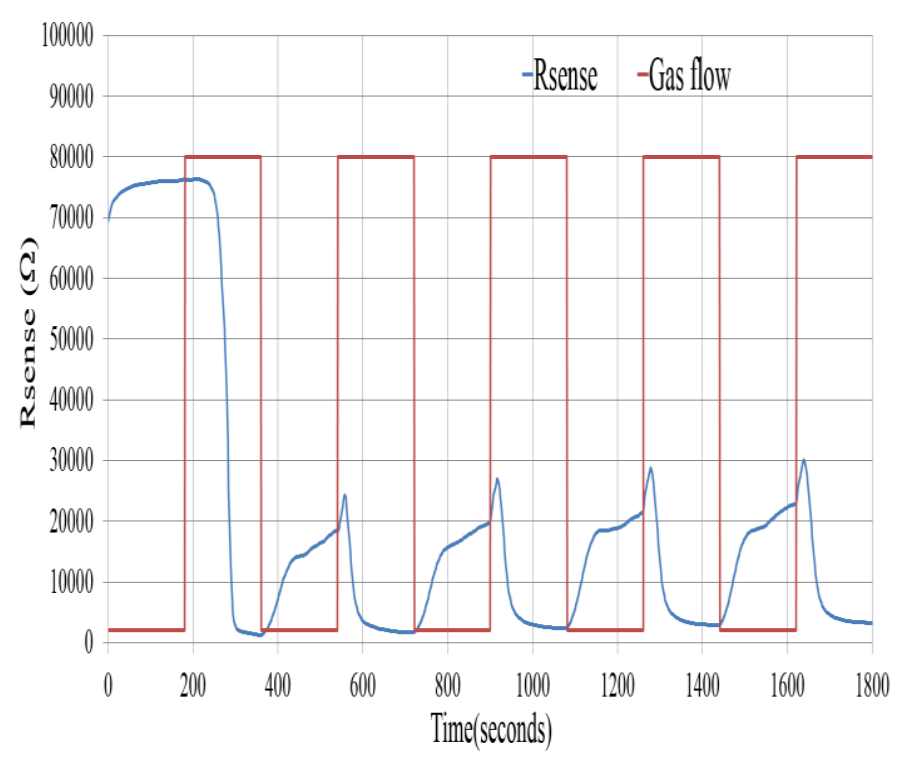

Fig. 4: Response of the sensor for the $5.5 \mathrm{ml}$ solvent of ethyl alcohol.
There is a delayed response of the sensor in the first cycle as compared to the succeeding cycles. There is also an abrupt increase of response signal when the gas switch is turned on. This change happened due to the slight change in pressure in the opposite direction when the switch was turned on. Due to the delayed response of the sensor with this suspension, the total duration of the experiment was continued till 1800 seconds, unlike the following durations with different dispersion mediums that continued till 3600 seconds.

\section{Experiment with $2 \mathrm{gm} \mathrm{SnO}_{2}+6 \mathrm{ml} \mathrm{Et-OH}$}

The response of the coated interdigital sensor for the dispersion medium of $6 \mathrm{ml}$ of ethyl alcohol is shown in figure 5 . The response of the sensor with this coating is much better compared to the preceding suspension as shown in figure 4. The response time for the gas-on condition was around 20 seconds and was around 24 seconds for the gas-off condition. The consistency of sensitivity values was checked by increasing the experimental duration to 3600 seconds. It is seen that the change in response signal values is almost same for all the cycles, expect the first one. 


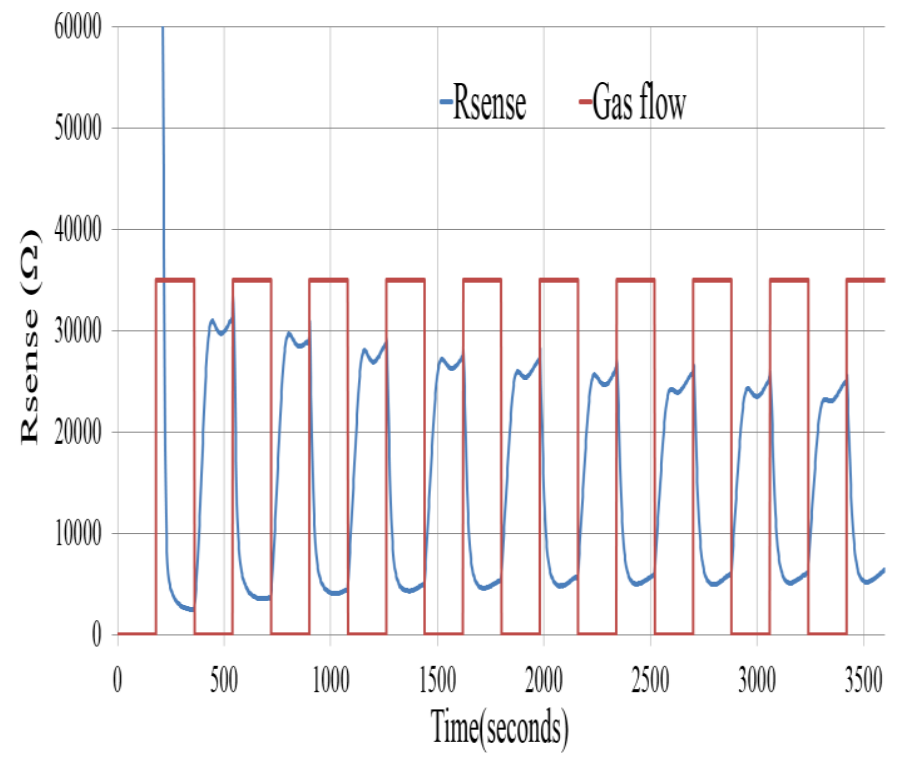

Fig. 5: Response of the sensor for the $6 \mathrm{ml}$ solvent of ethyl alcohol.

\section{Experiment with $2 \mathrm{gm} \mathrm{SnO}_{2}+6.5 \mathrm{ml} \mathrm{Et-OH}$}

The next experiment conducted was the sensing of LPG using a coated layer of $6.5 \mathrm{ml}$ of ethyl alcohol as a dispersion medium. The response is shown in figure 6 . It is seen from the graph that the response in this medium is better than $5.5 \mathrm{ml}$ of ethyl alcohol but has a delayed response compared to the preceding dispersion medium. This medium has an intermediate response time compared to the other two tested solutions. The response time for the gas-on condition was around 35 seconds, where for the gas-off condition was around 30 seconds. The spike that happened during the switching on of the gas is present prominently in this medium too.

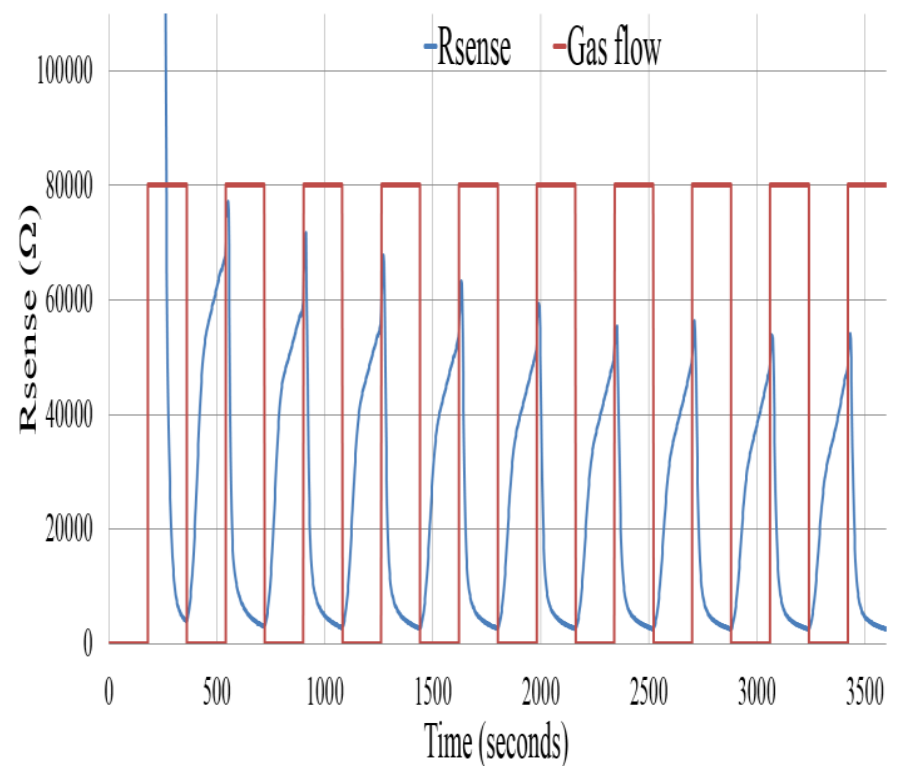

Fig. 6: Response of the sensors for the $6.5 \mathrm{ml}$ solvent of ethyl alcohol.

\section{Experiment with 2 gm $\mathrm{SnO}_{2}+7 \mathrm{ml} \mathrm{Et-OH}$}

The response of the coated interdigital sensor to the dispersion medium of $7 \mathrm{ml}$ of ethyl alcohol is shown in figure 7 . The decrease and increase of response the change of concentration of gas had a mixed response to $6 \mathrm{ml}$ and $6.5 \mathrm{ml}$ of ethyl alcohol. Similar to the preceding results, the response time for the gas-on condition for the first cycle was different from the other ones with this dispersion medium. It was around 28 seconds for gas-on condition. The response time for the gasoff condition was similar to the result of the $6 \mathrm{ml}$ of ethyl alcohol which was around 26 seconds. Moreover, the spike that was present in the two suspensions, $2 \mathrm{gm}$ of $\mathrm{SnO}_{2}$ with $5.5 \mathrm{ml}$ and $6 \mathrm{ml}$ of ethyl alcohol respectively is not present in this solution.

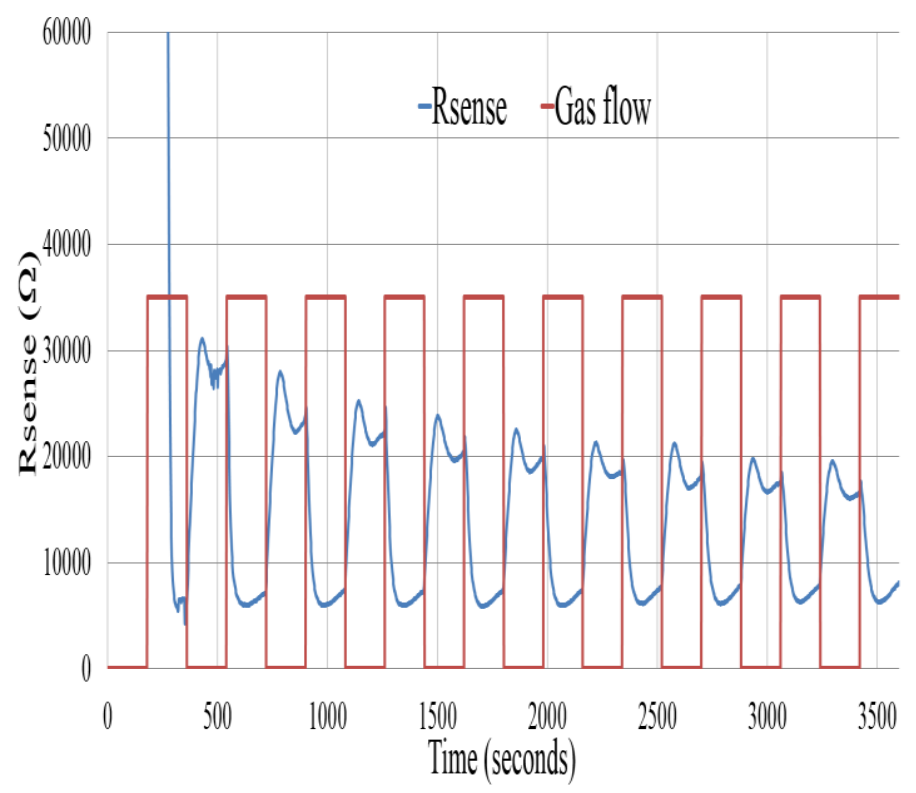

Fig. 7: Response of the sensor for the $7 \mathrm{ml}$ solvent of ethyl alcohol.

\section{Tin oxide $\left(\mathrm{SnO}_{2}\right)$ particle size optimization}

It is noted from the comparative study of the response of the coated interdigital sensor for the four suspensions shown in figures 4-7 that the optimum result was achieved with the suspension composed of $2 \mathrm{gm}$ of $\mathrm{SnO}_{2}$ with $6 \mathrm{ml}$ of ethyl alcohol. Once the concentration of this suspension was standardized, the particle size of $\mathrm{SnO}_{2}$ was varied by sieving in the next stage. Sieving was done on the dispersed phase to vary the sensitivity of the coated layer. A perforated membrane was used for the particles to sieve which was collected by a closed collection chamber below it. The size of the holes in the membrane was varied to determine the effect of specific sizes on sensitivity. Four different ranges of the hole sizes of the membrane were chosen for sieving: particles size between 75-63 microns, 63-53 microns, 53-45 microns and less than 45 microns. The tool was operated based on three parameters: power assigned to the tool for sieving, duration of sieving and the weight of the collected sieved 
material. The relation between the power assigned to the sieving tool and the weight of the output is shown in figure 8 . It is seen from the graph that the relation between the two chosen parameters is almost linear. Hence, the sieving of the particles was done on 880 watts to obtain the maximum sieved output. The following results, figures 9-12 show the response of the interdigital sensor with the coating of sieved particles with fixed suspension concentration.

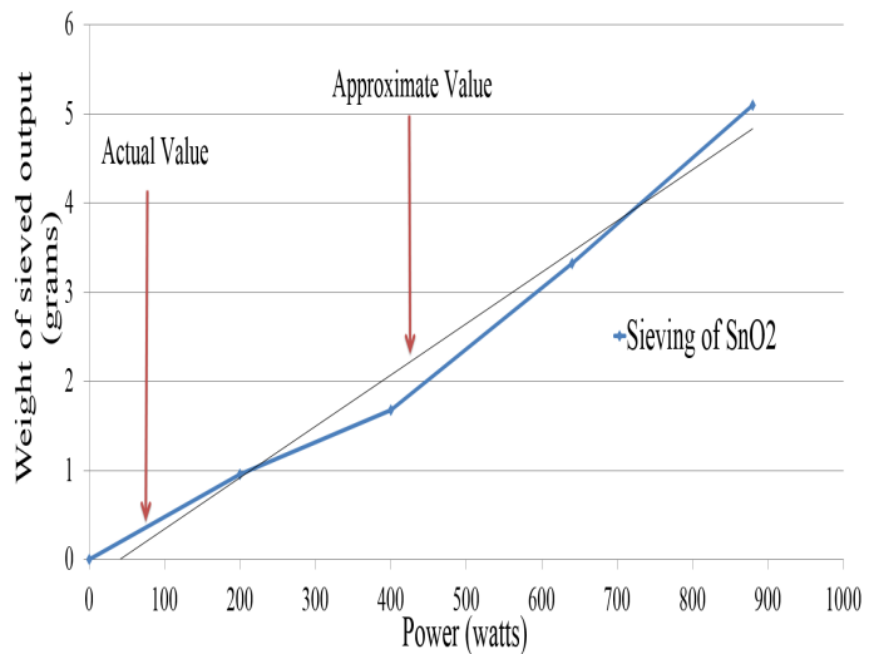

Fig. 8: Relation between power and weight in sieving tool.

\subsection{Experiment with $2 \mathrm{gm}+6 \mathrm{ml}$ Et-OH: $\mathrm{SnO}_{2}$ particle size between 75 and 63 microns}

The initial sieving was done for particle size between 75 and 63 microns. The response of the coated sensor with the suspension layer containing the sieved medium is shown in figure 9. It is seen from the graph, the response time to the gas is very low.

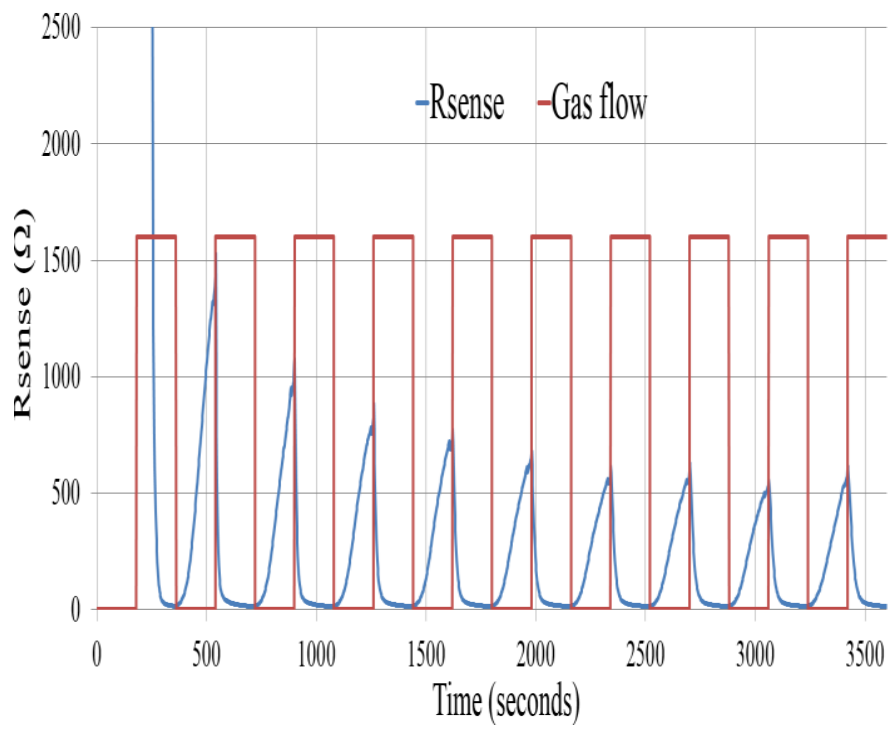

Fig. 9: Response of the sensor for sieving between 75 and 63 microns of the dispersed medium
Here the response time has subsequently increased from the preceding experiments done with different suspensions, but the change in response signal values is very low in every cycle. The change is not constant and the difference in the response of the sensor values from the initial cycle to the succeeding cycles is very large.

\subsection{Experiment with $2 \mathrm{gm}+6 \mathrm{ml}$ Et-OH: $\mathrm{SnO}_{2}$ particle size between 63 and 53 microns}

The next suspension is for the sieved particles between the range 63-53 microns and $6 \mathrm{ml}$ of ethyl alcohol. The response of the sensor is shown in figure 10. The response time for the gas-on condition is around 6 seconds and is 20 seconds for the gas-off condition. The response time for the initial cycle was delayed compared to the succeeding cycles and was around 10 seconds. There was also an abrupt change in signal readings during the initial cycle. This change was similar to the preceding readings done with different suspensions with the exception that the change was slightly more in this case. The response time for the gas-on condition was almost constant for every cycle.

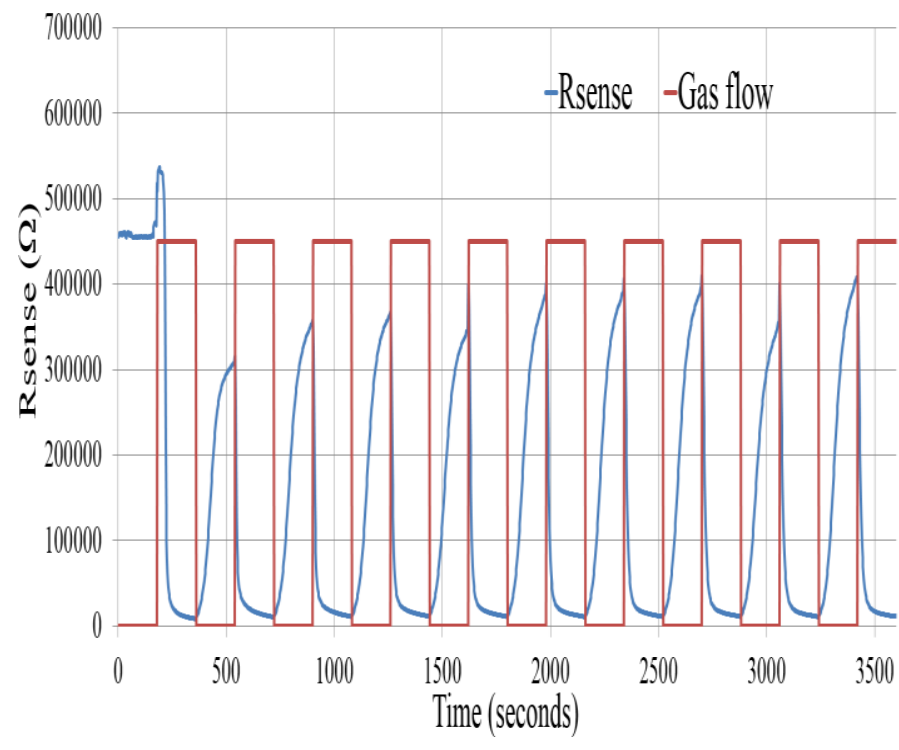

Fig. 10: Response of the sensor for between 63 and 53 microns of the dispersed medium.

\subsection{Experiment with $2 \mathrm{gm}+6 \mathrm{ml}$ Et-OH: $\mathrm{SnO}_{2}$ particle size between 53 and 45 microns}

Next is the suspension formed by $6 \mathrm{ml}$ of ethyl alcohol and sieved particles between 53 and 45 microns. The response time of this suspension is the best one compared to all the suspensions. The response is shown in figure 11. It is seen from the graph, the response for the gas-on condition is constant in all the cycles. The response time for the gas-on condition is around 4 seconds, but the response time for the gas-off condition is 18 seconds, which is similar to the previous suspension. The spike that was present in the response with other suspensions is absent here. 


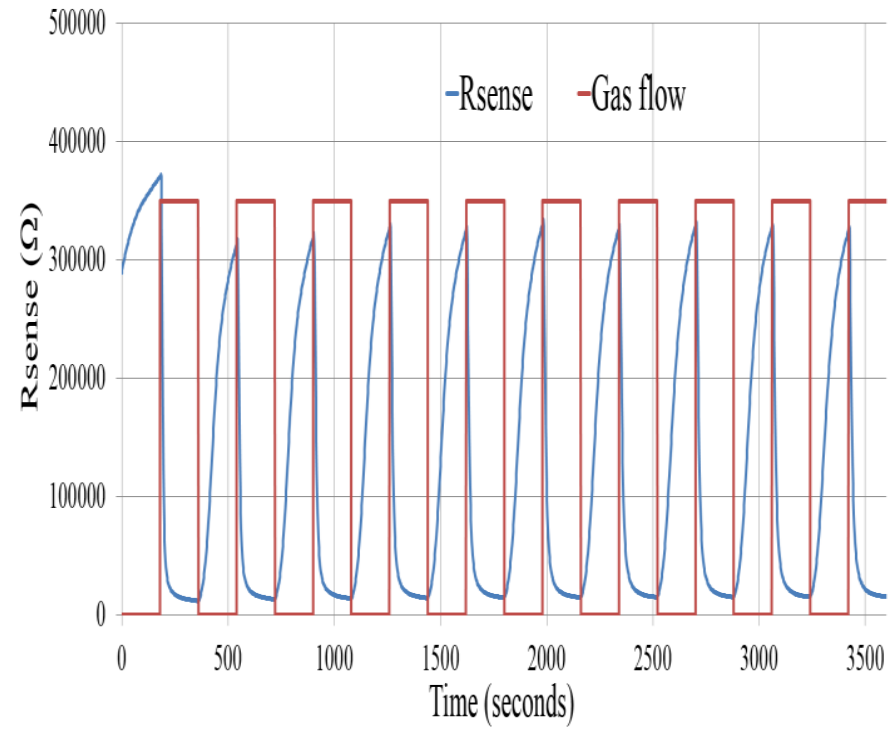

Fig. 11: Response of the sensor for sieving between 53 and 45 microns of the dispersed medium.

\subsection{Experiment with $2 \mathrm{gm}+6 \mathrm{ml}$ Et-OH: $\mathrm{SnO}_{2}$ particle size $<45$ microns}

The response of the coated interdigital sensor with the sieved dispersed medium for less than $\mathbf{4 5}$ microns is shown in figure 12. It is seen from the response that the delay in the response time of this suspension is almost similar to the response time of the sieved $\mathrm{SnO}_{2}$ particles between 53 and 45 microns.

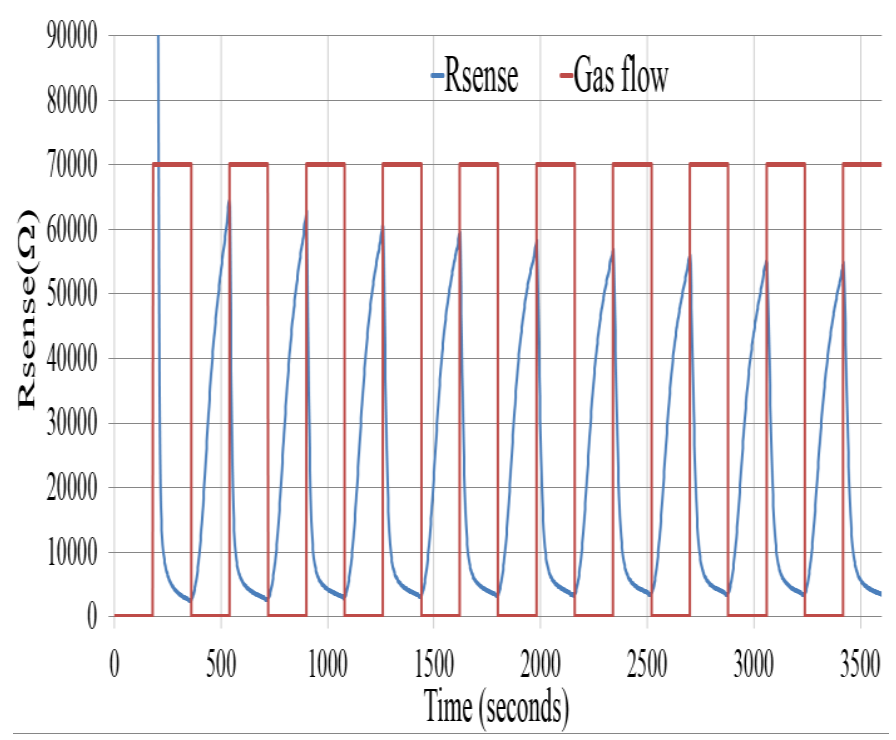

Fig. 12: Response of the sensor for sieving for less than 45 microns of the dispersed medium.

The response time for the gas-on condition was around 12 seconds, whereas it was around 22 seconds for the gas-off condition. The spike that was present to a certain extent in all the preceding tested suspensions was also absent here. The change was the inconsistency of the signal readings for gasonthe condition for every cycle compared to the initial cycle.

\subsection{Discussion}

The detection of LPG is due to chemisorption of oxygen to sensor coated with $\mathrm{SnO}_{2}$. The change in the response of flow of current with respect to the gas condition is shown in figure 13. The figure indicates the change in response signal, which takes place as a result of oxidation of LPG in ambient condition. The adsorption of LPG on the sensor coating causes the electron to attach with the surface oxygen ion of $\mathrm{SnO}_{2}$ leading to the flow of current.

The response of the sensor as a function of humidity at a controlled environment has been investigated and is shown in figure 14. Comparing figure 14 with figure 13 , it is seen that the level of response as shown in figure 13 is much higher than that of figure 14. Moreover, the change of humidity during the experiment with LPG did not change significantly.

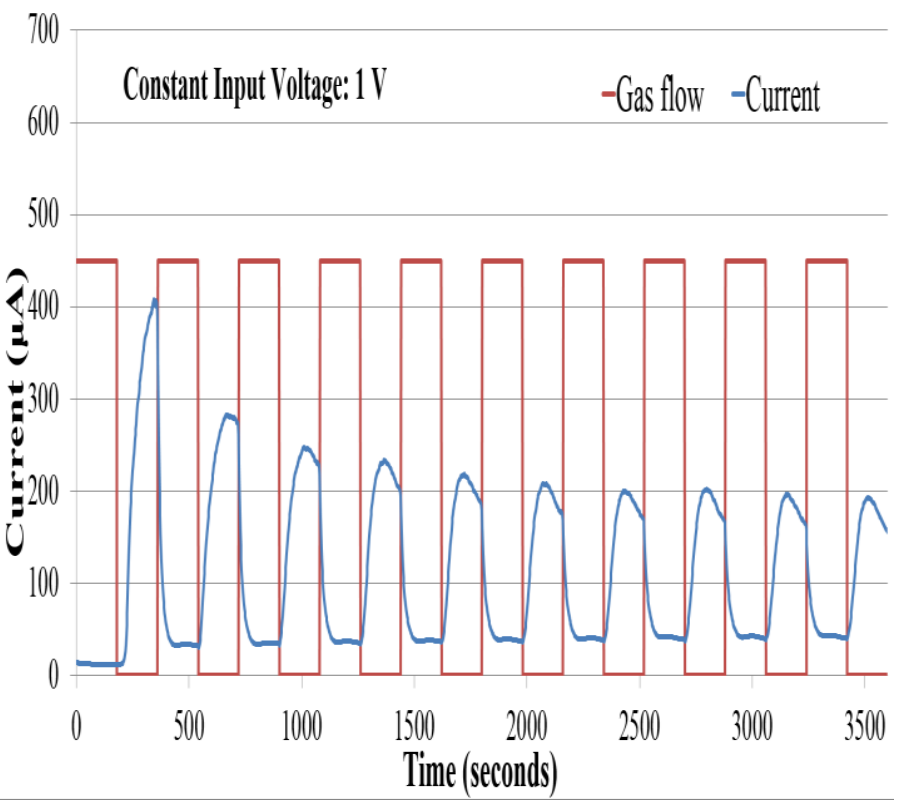

Fig. 13: Response of sensor for a change in current flow for $6 \mathrm{ml}$ of the dispersion medium.

It is concluded from the detailed experimentation that the response time of the coated interdigital sensors changes with the change in concentration of the suspension and size of the $\mathrm{SnO}_{2}$ particles. The experiments conducted on different suspensions on the sensor yielded different response time of the sensor towards the gas. The optimal response time in the increase and decrease of the value of response signal of the sensor takes place with dispersion medium of $6 \mathrm{ml}$ of ethyl alcohol with the $2 \mathrm{gm}$ of tin oxide. The variation in the size of the dispersed medium is another reason to cause a significant change in the response time. It could be seen from the experiments that the decrease in size of the grain particles proved to lead to a better response time. However, the size of these particles should not be decreased below a certain threshold level, as could be seen from the above experiments that the best results are obtained for the particle size between 53-45 microns. 


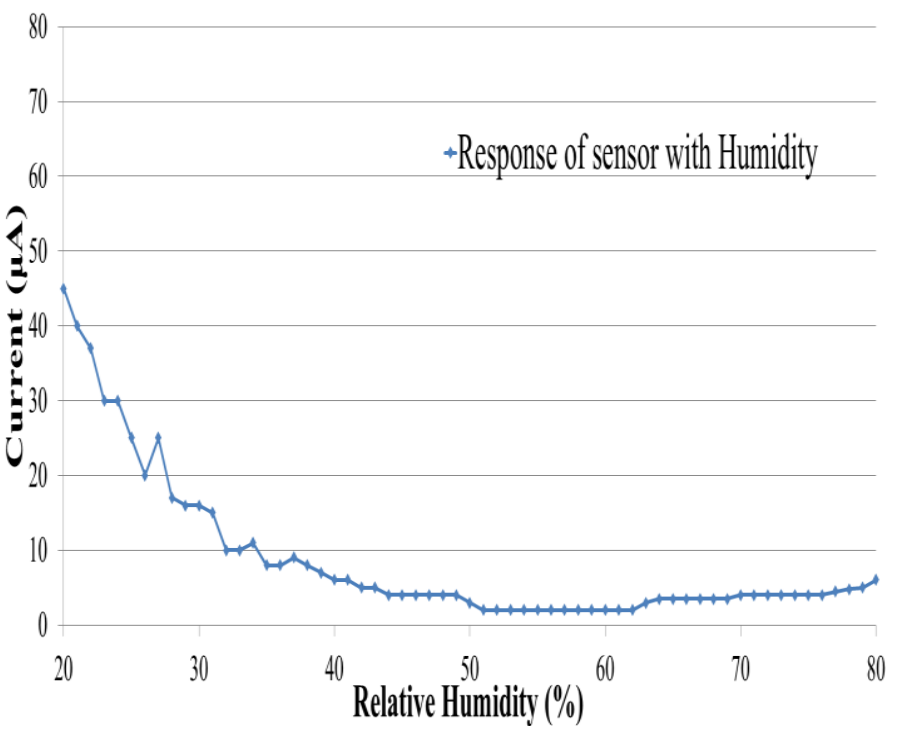

Fig. 14: The response of the sensor as a function of humidity in a controlled environment.

Some issues did arise during the experiments. The first one was the occurrence of sudden spikes, especially during the change in concentration of the gas. The probable reason could be the change in pressure that occurs on the sudden change in concentration. The difference in response time for the gas-off condition is another problem that took place during the experiments. This is possible due to the difference in kinetics of the adsorbed electrons from the sensing surface as a result of different suspension solutions. This can be addressed by setting a threshold value for the increase in impedance during the gas-off condition. The outlet of the electrochemical cell can also be improved for quick liberate of the trapped gas molecules. Another issue was the range of change in the response signal levels between the first cycle compared to the succeeding cycles in every suspension. Two reasons can be possible for this phenomenon. One would be the presence of adsorbed molecules of LPG after the first cycle. When the gas switch was turned on, the coated sensor absorbed the gas molecules. When the gas switch was turned off after 3 minutes, all the adsorbed gas molecules could not be released from the sensor. Due to this, the response signal value of the sensor would not rise above a certain level. The second reason would be the insufficient time of the cycle that did not allow the response signal value to rise, before turning to the next cycle.

\section{MICROCONTROLLER BASED SENSING SYSTEM}

The next step approach for the gas detection would be to develop a microcontroller based sensing system to make the proposed system for practical application. The fabricated sensor has been interfaced to an Arduino based microcontroller system to make it a complete sensing system. Figure 15 and 16 shows the schematic diagram and necessary components used in the system. The microcontroller measures both the magnitude of the sensing voltage as well as the phase angle between the input voltage and the sensing voltage.
Experiments have been conducted to test the developed system. Table 3 shows the results obtained from experiments. The output voltage across the sensing resistance increases with the increase of concentration of gas. The system has been designed to give a full scale voltage at 20,000 ppm.

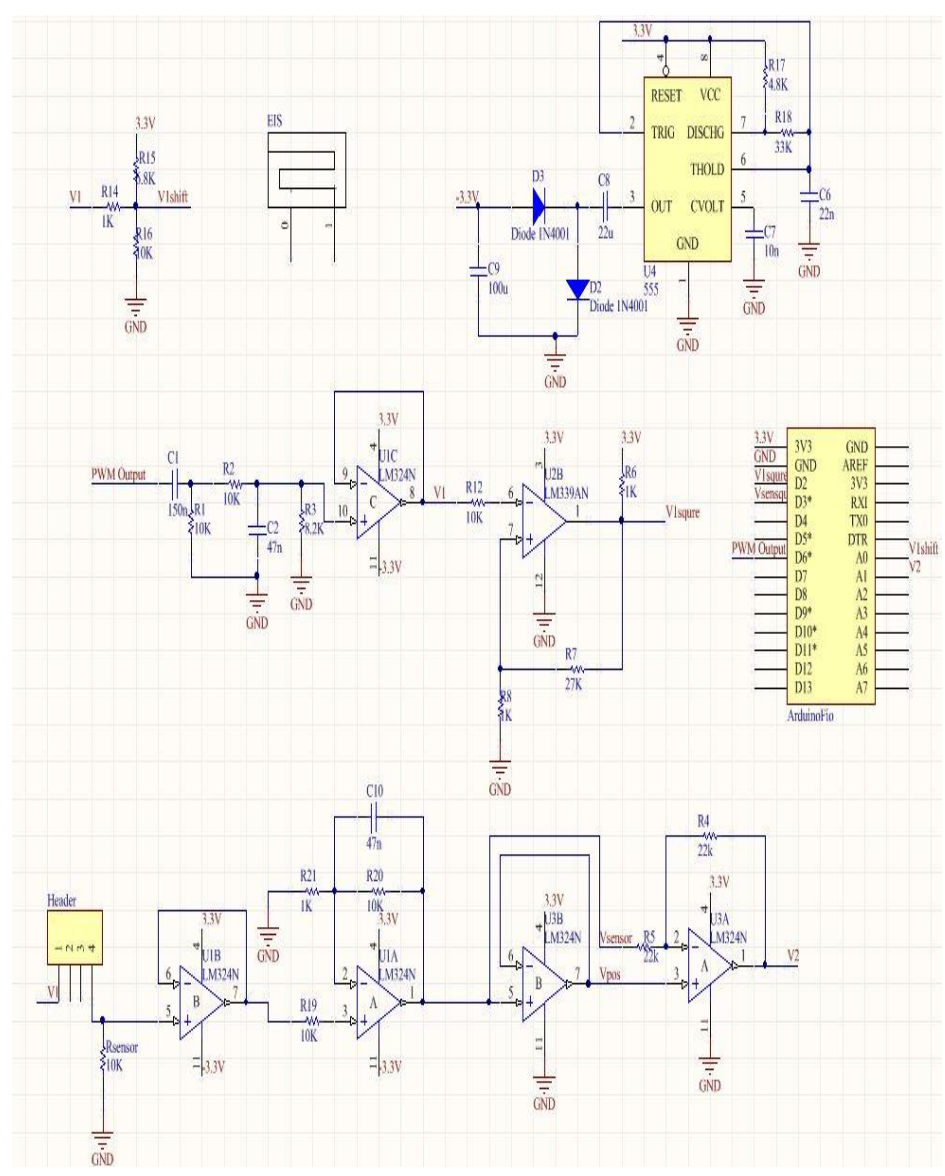

Fig. 15: Schematic diagram for microcontroller based system for gas sensing.

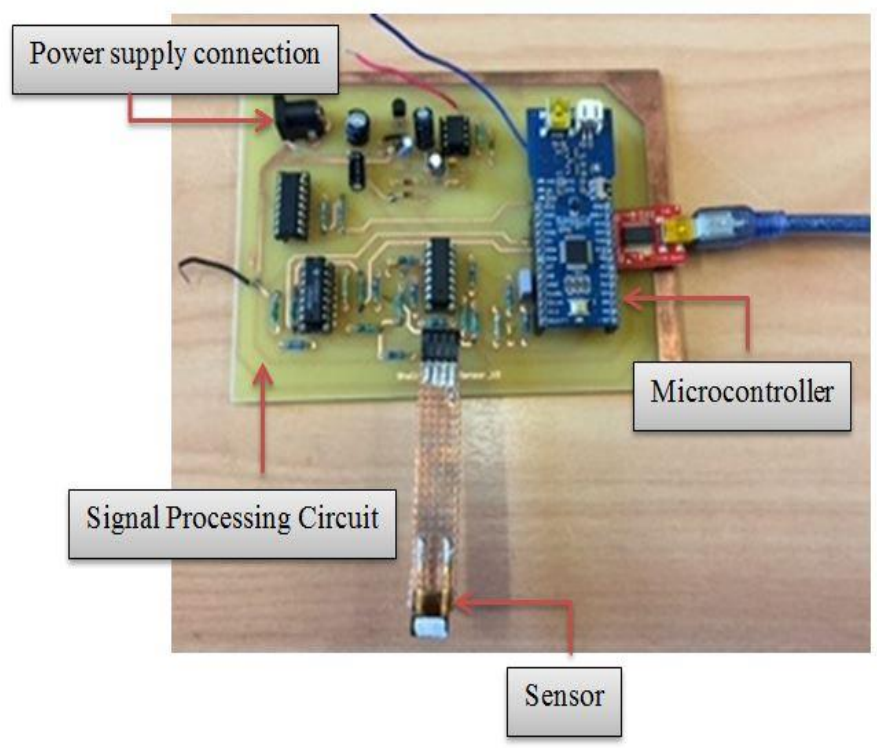

Fig. 16: Components used in gas sensing system. 
Table 3: Results of microcontroller based gas sensing system

\begin{tabular}{|c|c|c|}
\hline Gas conc. $(\mathrm{ppm})$ & $\mathrm{V}_{\text {sense }}(\mathrm{V})$ & $\begin{array}{c}\text { Phase angle }(\Phi) \\
\text { (degrees) }\end{array}$ \\
\hline 0 & 0 & 0 \\
\hline 500 & 0.32 & 2.7 \\
\hline 1000 & 0.63 & 3.6 \\
\hline 2000 & 1.07 & 10.6 \\
\hline 5000 & 1.34 & 13.5 \\
\hline
\end{tabular}

\section{CONCLUSION AND FUTURE WORK}

The paper represents the work done on the effects of varying the particle size, concentration of the dispersion medium on the response time to the gas. Different concentrations of the dispersion medium in the coated suspension have been tested to determine the accurate composition required to achieve the optimal sensitivity of $\mathrm{SnO}_{2}$ thin layer. Investigation of the particle size of the dispersed medium into four different ranges has played a key role to optimize the sensor's response time. The $\mathrm{SnO}_{2}$ particles have been suspended in the dispersion medium and spin-coated on the sensing surface of the interdigital sensors fabricated with gold electrodes on the surface of a single crystal Silicon substrate using MEMSbased fabrication techniques. The control over adsorption and desorption kinetics of the gas molecules in the coated layer has been achieved by reducing the particle size instead of exposing the sensor to high temperature. Ambient operation of the sensor has provided a novel feature of the developed technique that made it unique among its counterparts. The response time of the coated sensor has been encouraging and owns a promising potential for the development of a more efficient gas sensing system. In future, wireless transceiver would be included to make a smart sensing system to detect leakage of gas.

\section{REFERENCES}

[1] J. Stawczyk, "Experimental evaluation of LPG tank explosion hazards," Journal of hazardous materials, vol. 96, pp. 189-200, 2003.

[2] R. E. Melchers and W. R. Feutrill, "Risk assessment of LPG automotive refuelling facilities," Reliability Engineering \& System Safety, vol. 74, pp. 283-290, 2001.

[3] C. Raiyani, S. Shah, N. Desai, K. Venkaiah, J. Patel, D. Parikh, et $a l$., "Characterization and problems of indoor pollution due to cooking stove smoke," Atmospheric Environment. Part A. General Topics, vol. 27, pp. 1643-1655, 1993.

[4] C.-H. Lai, C.-C. Chang, C.-H. Wang, M. Shao, Y. Zhang, and J.-L. Wang, "Emissions of liquefied petroleum gas (LPG) from motor vehicles," Atmospheric Environment, vol. 43, pp. 1456-1463, 2009.

[5] L. Dong, Z. Cui, and Z. Zhang, "Gas sensing properties of nano$\mathrm{ZnO}$ prepared by arc plasma method," Nanostructured materials, vol. 8, pp. 815-823, 1997.

[6] A. Bodade, A. Bende, and G. Chaudhari, "Synthesis and characterization of CdO-doped nanocrystalline $\mathrm{ZnO}$ : $\mathrm{TiO}$ 2-based H 2 S gas sensor," Vacuum, vol. 82, pp. 588-593, 2008.

[7] T. Chen, Q. Liu, Z. Zhou, and Y. Wang, "A high sensitivity gas sensor for formaldehyde based on $\mathrm{CdO}$ and $\mathrm{In} 2 \mathrm{O} 3$ doped nanocrystalline SnO2," Nanotechnology, vol. 19, p. 095506, 2008.
[8] T. Chen, Z. Zhou, and Y. Wang, "Effects of calcining temperature on the phase structure and the formaldehyde gas sensing properties of CdO-mixed In $2 \mathrm{O}$ 3," Sensors and Actuators B: Chemical, vol. 135, pp. 219-223, 2008.

[9] X. Liu, Z. Xu, Y. Liu, and Y. Shen, "A novel high performance ethanol gas sensor based on $\mathrm{CdO} \square \mathrm{Fe} 2 \mathrm{O} 3$ semiconducting materials," Sensors and Actuators B: Chemical, vol. 52, pp. 270273, 1998.

[10] E. Leite, I. Weber, E. Longo, and J. A. Varela, "A new method to control particle size and particle size distribution of $\mathrm{SnO} 2$ nanoparticles for gas sensor applications," Advanced Materials, vol. 12, pp. 965-968, 2000.

[11] A. Kolmakov, D. Klenov, Y. Lilach, S. Stemmer, and M. Moskovits, "Enhanced gas sensing by individual $\mathrm{SnO} 2$ nanowires and nanobelts functionalized with Pd catalyst particles," Nano Letters, vol. 5, pp. 667-673, 2005.

[12] N. Barsan, M. Schweizer-Berberich, and W. Göpel, "Fundamental and practical aspects in the design of nanoscaled $\mathrm{SnO} 2$ gas sensors: a status report," Fresenius' journal of analytical chemistry, vol. 365, pp. 287-304, 1999.

[13] H.-C. Chiu and C.-S. Yeh, "Hydrothermal synthesis of $\mathrm{SnO} 2$ nanoparticles and their gas-sensing of alcohol," The Journal of Physical Chemistry C, vol. 111, pp. 7256-7259, 2007.

[14] S. Munnix and M. Schmeits, "Electronic structure of tin dioxide surfaces," Physical Review B, vol. 27, p. 7624, 1983.

[15] A. I. Zia, A. Mohd Syaifudin, S. Mukhopadhyay, I. Al-Bahadly, P. $\mathrm{Yu}, \mathrm{C}$. Gooneratne, et al., "Development of Electrochemical Impedance Spectroscopy based sensing system for DEHP detection," in Sensing Technology (ICST), 2011 Fifth International Conference on, 2011, pp. 666-674.

[16] A. I. Zia, A. Mohd Syaifudin, S. Mukhopadhyay, P. Yu, I. AlBahadly, J. Kosel, et al., "Sensor and instrumentation for progesterone detection," in Instrumentation and Measurement Technology Conference (I2MTC), 2012 IEEE International, 2012, pp. 1220-1225.

[17] A. I. Zia, S. Mukhopadhyay, I. Al-Bahadly, P. Yu, C. P. Gooneratne, and J. Kosel, "Introducing molecular selectivity in rapid impedimetric sensing of phthalates," in Instrumentation and Measurement Technology Conference (I2MTC) Proceedings, 2014 IEEE International, 2014, pp. 838-843.

[18] A. I. Zia, M. S. A. Rahman, S. C. Mukhopadhyay, P.-L. Yu, I. AlBahadly, C. P. Gooneratne, et al., "Technique for rapid detection of phthalates in water and beverages," Journal of Food Engineering, vol. 116, pp. 515-523, 2013.

[19] A. Mohd Syaifudin, S. Mukhopadhyay, and P. Yu, "Electromagnetic field computation using COMSOL Multiphysics to evaluate the performance of novel interdigital sensors," in Applied Electromagnetics Conference (AEMC), 2009, 2009, pp. 14.

[20] A. Mohd Syaifudin, S. Mukhopadhyay, and P. Yu, "Modelling and fabrication of optimum structure of novel interdigital sensors for food inspection," International Journal of Numerical Modelling: Electronic Networks, Devices and Fields, vol. 25, pp. 64-81, 2012.

[21] A. Mohd Syaifudin, M. Yunus, S. Mukhopadhyay, and K. Jayasundera, "A novel planar interdigital sensor for environmental monitoring," in Sensors, 2009 IEEE, 2009, pp. 105-110.

[22] A. Rahman and M. S. Bin, "Novel planar interdigital sensors for the detection of bacterial endotoxins: a thesis presented in partial fulfilment of the requirements for the degree of Doctor of Philososphy in Electronics Engineering at Massey University, Palmerston North, New Zealand," 2012.

[23] M. S. A. Rahman, S. C. Mukhopadhyay, and P.-L. Yu, "Novel Planar Interdigital Sensors," in Novel Sensors for Food Inspection: Modelling, Fabrication and Experimentation, ed: Springer, 2014, pp. 11-35.

Authors' biographies are available in Part I, Sensors-13325-2015. 